\title{
Feasibility of a Six Week High Intensity Interval Training Program in Patients with Non- Small Cell Lung Cancer: A Randomized Pilot Study
}

\author{
Emily Walsh ${ }^{1,2}$, Guy Hajj-Boutros ${ }^{3}$, Guillaume Bastarache ${ }^{1}$, Andréa Faust ${ }^{3}$ and Alain-Steve Comtois ${ }^{1^{*}}$ \\ ${ }^{1}$ Department of Exercise Science, Université du Québec à Montréal, Canada \\ ${ }^{2}$ Loyola University, Chicago Stritch School of Medicine, USA \\ ${ }^{3}$ Research Institute of the McGill University Health Center (MUHC), Canada

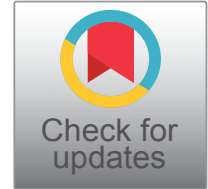 \\ *Corresponding author: Alain-Steve-Comtois, PhD, Department of Exercise Science Université du Québec à Montréal, C.P. \\ 8888, Succursale Centre-Ville Montreal, Quebec, H3C 3P8, Canada, Tel: 001-514-987-3000 ext. 1506, Fax: 001-514-987- \\ 6616
}

\section{Abstract}

High Intensity Interval Training (HIIT) has become a mainstream, frequently investigated form of exercise training and has been used as a modality for physical activity in cancer patients. The objective of this study was to determine the feasibility of advanced Non-Small Cell Lung Cancer (NSCLC) patients performing a program of HIIT. To our knowledge, this is the first study to investigate a HIIT program for these patients. Ten participants were recruited for this study. Five were controls and five were in the intervention group. The HIIT program took place twice a week for six weeks. Measurements of physical fitness were determined at baseline and six weeks (six-minute walk test, musculoskeletal tests and a balance test). HIIT was performed on an ergocycle. Training consisted of a five-minute warm up, followed by repeated intervals of 10 seconds of high intensity and 60 seconds of low intensity, ending with a three-minute cool down. Rating of Perceived Exertion (RPE) was measured using the Borg scale. During HIIT, participants progressed in the number of intervals performed. Intensity as measured in watts more than doubled during the program. The RPE did not significantly change. Advanced NSCLC patients are able to do HIIT. They show progression in intensity and number of intervals. RPE was maintained, suggesting that participants were capable of maintaining an elevated exercise intensity at every training session. Results show that HIIT produces physiological improvements. Thus, HIIT is feasible and is well received as a training modality by advanced NSCLC patients. It is safe with good compliance, and more interestingly demonstrated muscle power improvement in a short six-week period of intervention.
\end{abstract}

\section{Keywords}

HIIT, Non-small cell lung cancer, Exercise

\section{Objectives}

Lung cancer is the second most common cancer in men and women. 235,760 people are estimated to be diagnosed with lung cancer in the United States in 2021 with the majority (84\%) of those being NonSmall Cell Lung Cancer (NSCLC). It is projected that in $2021,131,880$ people will die from lung cancer, which represents $21.7 \%$ of total cancer deaths [1]. Although death rates have been decreasing, the 5 year survival rates for lung cancer patients continue to be low at $21 \%$. NSCLC has a higher 5 year survival rate than small cell lung cancer at $25 \%$ vs. $7 \%$ [2].

Currently there is support for the role of physical activity in reducing the risk of cancer development and in reducing cancer-related mortality rates for different types of cancer including lung cancer $[3,4]$. Several studies have shown that physical activity leads to a significant reduction in the symptom burden of cancer patients. Physical activity has been shown to help control cancer-related symptoms during and after chemotherapy [5]. In particular, physical activity has been found to benefit patients' quality of life,

\section{short six-week period of intervention.}

Citation: Walsh E, Boutros GH, Bastarache G, Faust A, Comtois AS (2021) Feasibility of a Six Week High Intensity Interval Training Program in Patients with Non- Small Cell Lung Cancer: A Randomized Pilot Study. Int J Sports Exerc Med 7:200. doi.org/10.23937/2469-5718/1510200

Accepted: September 20, 2021; Published: September 22, 2021

Copyright: (C) 2021 Walsh E, et al. This is an open-access article distributed under the terms of the Creative Commons Attribution License, which permits unrestricted use, distribution, and reproduction in any medium, provided the original author and source are credited. 
functional capacity, length of hospital stay, strength and psychosocial and cognitive functions $[6,7]$.

Due to the nature of the disease, many patients with NSCLC often do not partake in physical activity. Granger, et al. 2014 compared 50 patients with stage I to IIIB NSCLC with 35 people who were healthy and similar in age. It was found that patients with NSCLC were significantly less active than healthy people similar in age. Patients with NSCLC spent less time outside, had higher depression scores, were less motivated, especially to exercise, and watched significantly more television compared to the control group [8].

High Intensity Interval Training (HIIT) involves repeated intervals of high intensity, meaning at an intensity greater than the anaerobic threshold, followed by periods of low intensity recovery. The idea behind this type of training is that it allows the participant performing the exercise to spend more time in high intensity zones [9]. This allows more improvement in endurance performance and physiological adaptations than traditional continuous exercise. It has been found to be especially beneficial in sedentary and recreationally active individuals $[9,10]$.

Studies have shown benefits of HIIT in cancer patients. A meta-analysis by Wallen, et al. showed that in cancer patients and survivors, HIIT lead to a significant effect on VO2 when compared to usual care. The authors also showed that HIIT can be safely performed by both cancer patients and survivors [11]. Adams, et al. found that in testicular cancer survivors, a 12-week HIIT program lead to improvements in cancer related fatigue, self-esteem, and both mental and physical health related quality of life. Their study had almost $100 \%$ adherence to the HIIT protocol, which shows the feasibility and tolerability as well as the time-efficiency of HIIT [12]. A study by Mijwel, et al. 2018 showed that in women with stage I-IIIA breast cancer planning to receive chemotherapy, those that performed HIIT showed a decreased symptom burden and increased scores on health quality of life questionnaires [13].
Licker, et al. hypothesized that having preoperative lung cancer patients perform HIIT would decrease their post-surgical complications. Although no improvement in post-surgical complications was discovered, their patients did have an increase in aerobic capacity. It also showed the feasibility of performing HIIT on lung cancer patients, as no severe adverse effects were observed during the study [14]. Thus, it appears that HIIT can be well sustained by cancer patients and leads to improvements in well-being.

The primary objective of this study was to evaluate the feasibility and the impact of a six-week HIIT program on patients with advanced NSCLC (stage IIIB or IV) and compare it to those who did not receive intervention. The hypothesis was that HIIT would be feasible for NSCLC patients.

\section{Methods}

\section{Subjects}

The study group consisted of ten patients, aged 4375 (62.5 \pm 13.3 years-old), with a diagnosis of stage IIIB $(N=1)$ or IV $(N=9)$ NSCLC. Demographics can be seen in Table 1. Patients were recruited to participate in the study at the Hotel Dieu hospital in Montréal, Quebec, Canada. Every participant was receiving cancer treatment at Centre Hospitalier de l'Université de Montréal (CHUM). Patients were undergoing treatment with either chemotherapy ( $\mathrm{N}=3$ ) or immunotherapy $(\mathrm{N}=7)$ at the time. Informed consent was obtained, and participants were assigned to the intervention group or control group. Upon arrival at CHUM, each subject received an explanation about the purpose and procedures involved in the study and were given the opportunity to ask questions. The project was approved by the CHUM Research Ethics Committee.

Participants in both groups were given the same pamphlet about the importance of exercise in cancer patients and that encouraged physical activity to relieve the symptoms of advanced cancer. General physical activity recommendations consisted of walking or

Table 1: Demographics participant demographics in HIIT and control groups.

\begin{tabular}{|l|l|l|l|}
\hline Variables & HIIT $(\mathbf{n}=\mathbf{5})$ & Control (n = 5) & p \\
\hline Age (year) & $68.8 \pm 8.2$ & $56.2 \pm 15.2$ & 0.141 \\
\hline Male & 4 & 1 & \\
\hline Female & 1 & 4 & 0.66 \\
\hline Height $(\mathrm{cm})$ & 167.2 & 163.5 & 0.08 \\
\hline Weight $(\mathrm{kg})$ & 86.58 & 63 & 0.03 \\
\hline BMI & $30.8 \pm 4.8$ & $23.3 \pm 4.5$ & \\
\hline Immunotherapy & 1 & 3 & \\
\hline Chemotherapy & 1 & 2 & \\
\hline Surgery related to cancer & 1 & 2 & \\
\hline Hypertension & 2 & 0 & \\
\hline COPD & 1 & 0 & \\
\hline
\end{tabular}


cycling at moderate intensity for $\mathbf{3 0}$ minutes a day, in one or more session. Recommendations also included a variety of simple home-based strength and mobility exercises. Kinesiologists made themselves available by phone if participants had any questions regarding the physical activity recommendations.

Many of the participants had not been physically active in the past few months. The stress of HIIT may carry cardiac risk in patients with underlying coronary artery disease [15]. Therefore, all participants were required to be cleared by their physician to partake in HIIT prior to testing and training.

\section{Pre and Post-testing}

All subjects underwent: $A$ : $A$ six-minute walk test representing functional capacity, B: Musculoskeletal tests representing strength, C: A balance test. The subjects in the intervention group followed a 6-week HIIT program that took place twice a week, totaling 12 sessions.

Six-Minute Walk Test (6MWT): The 6MWT followed the guidelines outlined by the American Thoracic Society [16]. It was performed in a 30-meter non-carpeted marked corridor indoors at the CHUM. A 6 to 20 Borg Scale was used to measure the level of perceived effort at 2, 4 and 6 minutes [17]. Heart rate and blood pressure were recorded before and after the test. The 6MWT is favored for use in measuring changes due to interventions in patients with moderate to severe heart or lung disease so we found it suitable for our project testing advanced NSCLC patients [16].

Muscle strength: Two tests were used to assess strength. Handgrip strength was measured using a handgrip dynamometer (Jamar, Chicago, IL). Each participant was allowed three tries on each hand and the best of each was used [18]. The second test was a 20 second sit-to-stand test. The maximum number of repetitions completed in 20 seconds was recorded and perceived effort was once again measured using a 6 to 20 Borg scale. The same chair was used for every test for every participant [19].

Intervention group training: Training took place twice a week and consisted of a HIIT program. Training sessions were supervised by a kinesiologist with a maximum of two participants at a time. Participants were prohibited from training the day after receiving treatment. HIIT was performed on a standard stationary bicycle (Ergomatic 818 and 828E, Monark, Sweden) for safety and feasibility reasons. With intervals of HIIT initially lasting 10 seconds, the intervals were able to be better monitored and changed more quickly on a bike than they would have been on a treadmill or elliptical. Each participant was instructed on how to properly perform HIIT by a kinesiologist. There was a five-minute warm-up on the bike. Every participant started with 10 second intervals of high intensity followed by 60 seconds of a low intensity recovery period. High intensity was a minimum pedaling rate of 90 RPM. Low intensity was 50-60 RPM. This was repeated until the participant could no longer bike or reached a RPE of 17 . The Borg scale was used to assess effort level after each interval and was also used to determine when to stop training and when to increase the number of intervals. Because many of the patients were taking heart rate medications that could alter their heart rate, we used the Borg scale as an indication of effort and performance. A threeminute cool down was completed after the HIIT phase.

\section{Statistical analysis}

Results are reported as mean and standard deviation. Differences between groups were detected using a repeated measures two-factor (groups $\times$ time) ANOVA. When differences were detected a LSD post hoc analysis was performed to identify the significantly different pairs. Level of significance was set at $p<0.05$. Analysis was done using SPSS Version 24.

\section{Results}

Table 1 shows participant characteristics in the HIIT (4 men, 1 woman) and control (1 man, 4 women) groups. No significant differences were observed at baseline, except BMI. Among the 5 participants included in the HIIT group, 4 out of 5 attended all training sessions (12 sessions) of the 6 weeks program. No adverse events were reported during the exercise sessions. The one participant that did not complete the entire program did, however, attend 11 of 12 training sessions during the six-week period. Table 2 demonstrates patient's physical tests at baseline and 6 weeks.

HIIT protocol resulted in a marked improvement in the amount of power output (watts) across the intervention periods (session 1 to session 11 or 12). By the $4^{\text {th }}$ interval, a consistent improvement of more than $100 \%$ of the power output was observed. Interval 4 was used as it was the only interval completed by all participants starting from session 1 . This can be seen in Figure 1. Figure 2 shows the average total number of

Table 2: Physical test results at baseline and 6 weeks.

\begin{tabular}{|l|l|l|l|l|l|l|}
\hline Variables & HIIT $(\mathbf{n}=\mathbf{5})$ & Control $\mathbf{( n = 5 )}$ & & \\
\hline & Baseline & $\mathbf{6}$ weeks & Baseline & $\mathbf{6}$ weeks & Time effect & Group effect \\
\hline Handgrip Strength $(\mathrm{kg})$ & $68.4 \pm 21.8$ & $68.0 \pm 21.8$ & $58.9 \pm 32.4$ & $58.1 \pm 29.7$ & $p=0.34$ & $p=0.92$ \\
\hline Chair test (reps) & $9.9 \pm 7.3$ & $9.8 \pm 3.2$ & $10.2 \pm 6.6$ & $11.6 \pm 7.6$ & $p=0.04$ & $p=0.61$ \\
\hline 6MWT $(\mathrm{m})$ & $453.6 \pm 157$ & $466.4 \pm 164.3$ & $484.4 \pm 142.5$ & $494.3 \pm 173.2$ & $p=0.21$ & $p=0.95$ \\
\hline
\end{tabular}


Interval 4 Power Output vs. Session number

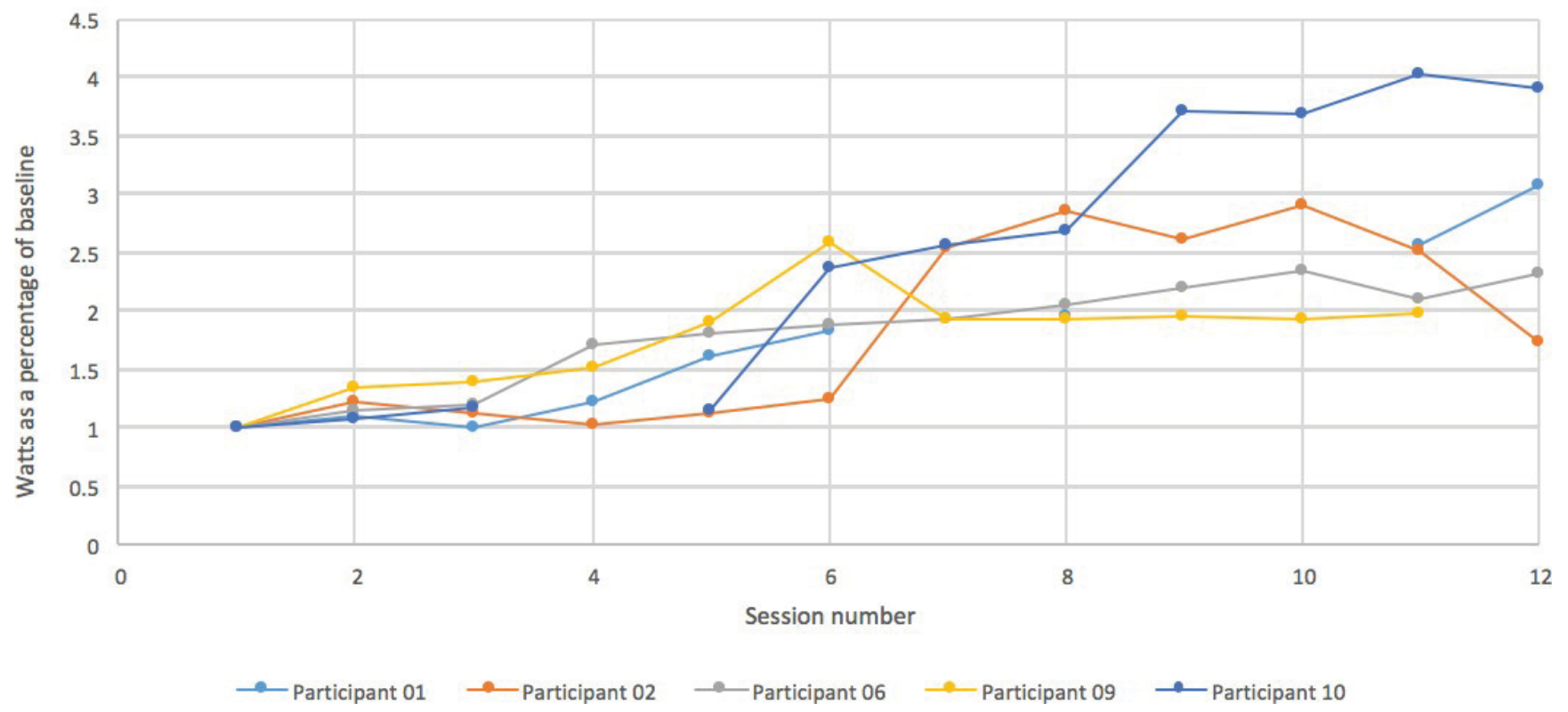

Figure 1: Interval 4 Power Output (watts) vs. Session Number.

\section{Intervals completed vs. session number}

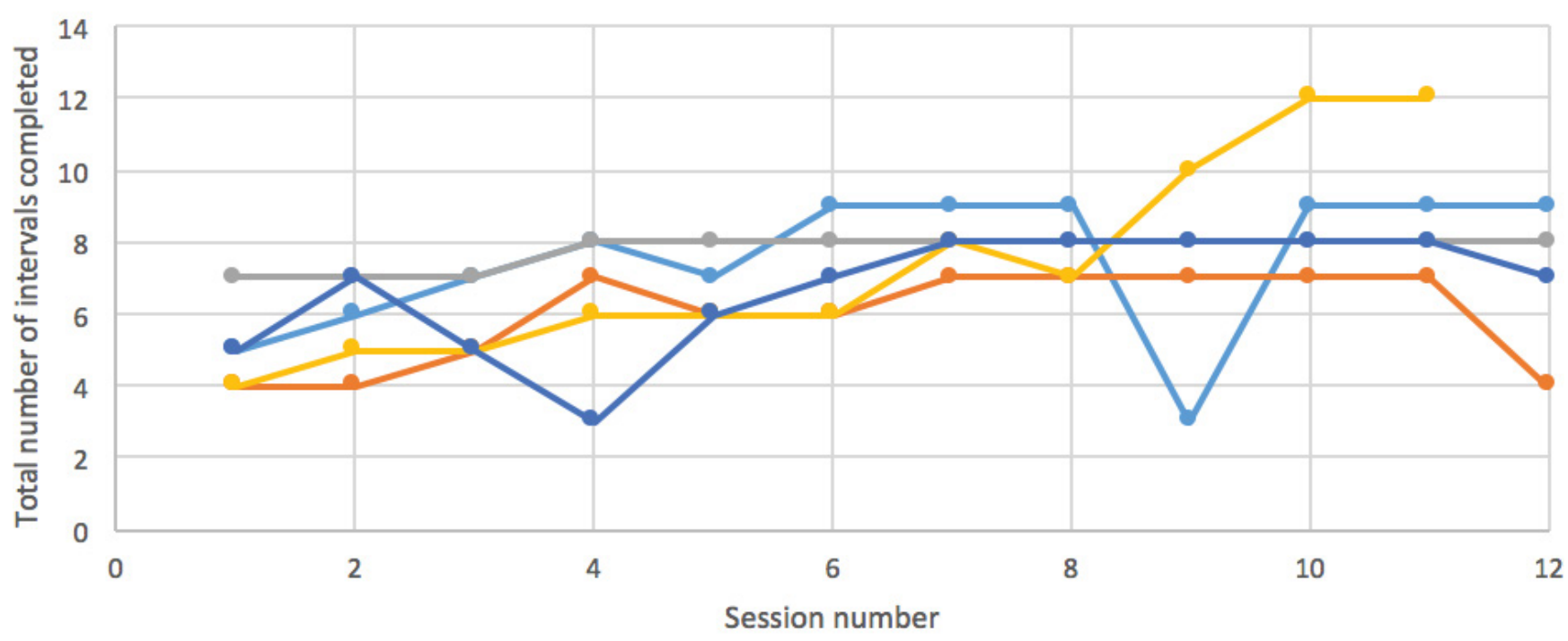

-Participant $01 \longrightarrow$ Participant $02 \longrightarrow$ Participant $06 \sim$ Participant $09 \sim$ Participant 10

Figure 2: Intervals completed vs. session number.

intervals increased from $5 \pm 1.2$ at session 1 to $8.8 \pm$ 1.92 at session 11 . Training volume was calculated by multiplying average power output (watts) per training session by 10 seconds (the length of the interval). Session 1 had an average of 780.4 watts seconds and session 11 had an average of 1980.9 watts*seconds, a 2.5-fold increase in training volume. Using the Borg scale, level of perceived exertion was determined during every interval, the warm-up and cool down. RPE stayed relatively constant throughout interval one $(11.4 \pm 3.29$ during session 1, $11.8 \pm 0.84$ during session 11 ). Interval 4 RPE varied slightly more from sessions 1 to 11 (15 \pm
$2.92,13.6 \pm 1.67)$. Average training volume compared to Borg scores (RPE) for each training session can be seen in Figure 3.

\section{Discussion}

The purpose of this pilot study was to evaluate the feasibility of a 6 week HIIT exercise program and compare it to a control group of patients with advanced NSCLC. This is the first study to examine the feasibility and effects of HIIT on these patients. We demonstrated that the implementation of only 12 sessions spanned over a six-week program of HIIT is feasible and beneficial 


\section{Average volume vs. RPE}

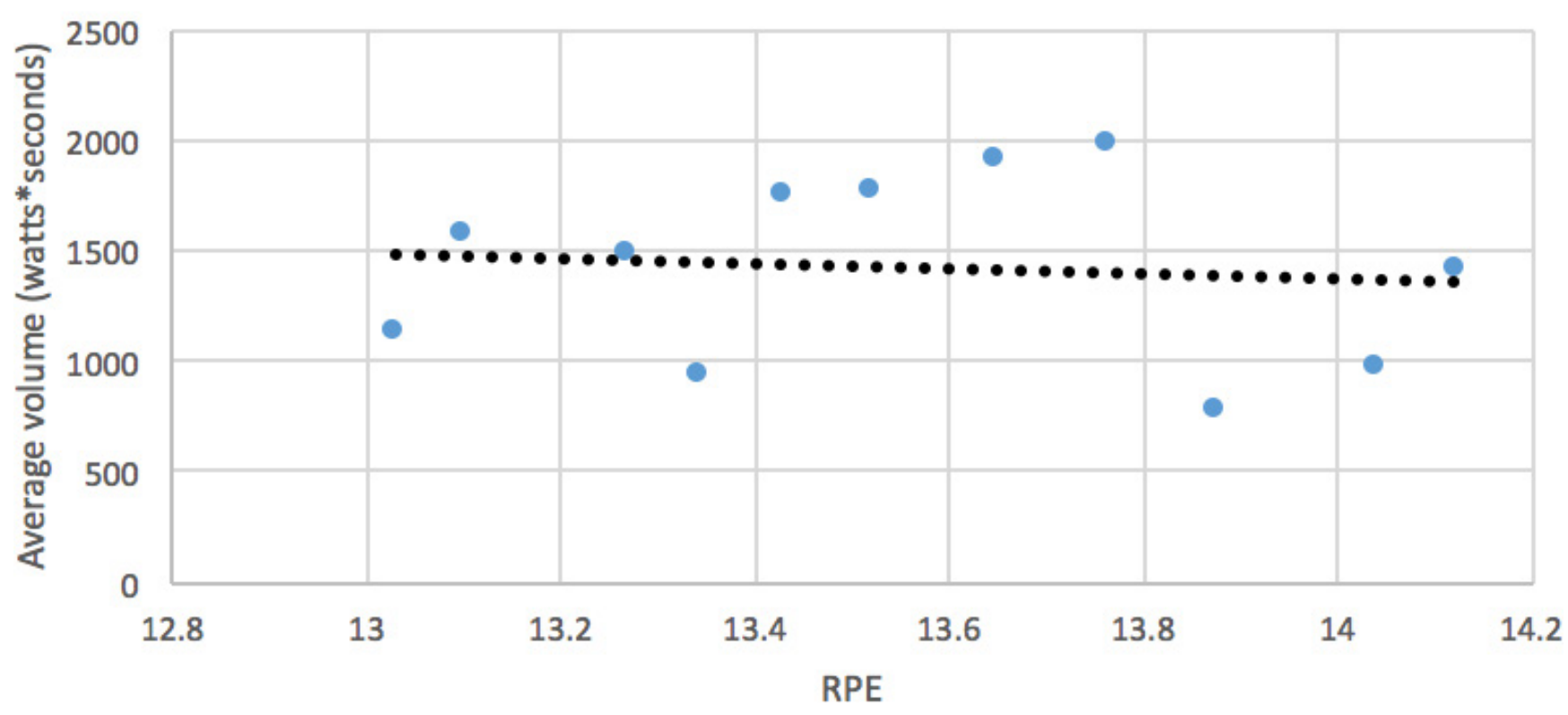

Figure 3: Average volume (watts*seconds) vs. RPE.

for advanced NSCLC patients. The drop-out rate was $0 \%$ and no adverse events were reported. Our results are similar to HIIT studies done in patients with heart failure, coronary artery disease, hypertension, obesity, and metabolic syndrome. These studies have found HIIT to be safe in these patients and to have more physiological benefits than moderate intensity continuous training $[20,21]$. Andersen, et al. proposed using a typical COPD rehabilitation program for lung cancer patients and found low adherence rates to exercise after finishing the exercise program. The authors cite that patients found their sessions repetitive and dull as reasons for lack of adherence [22]. HIIT could solve this problem as it has been found to be more interesting [23].

Similar to our results, other studies have also shown that exercise training was feasible in patients with NSCLC. In a randomized control study, patients with advanced NSCLC underwent an 8-week exercise program that took place three times a week. The training consisted of 30-40 minutes of training on a treadmill or ergocycle alternating between high intensity $\left(80 \% \mathrm{VO}_{2 \text { peak }}\right)$ and active recovery of moderate intensity $\left(60 \% \mathrm{VO}_{2 \text { peak }}\right)$. The control group received general care without supervised exercise training. Participants in the exercise group increased $\mathrm{VO}_{2 \text { peak }}$ and workload while the results in the control group remained unchanged. Both groups showed a significantly decreased fatigue level $(p=0.05 ; p=0.01)$ [24]. Temel, et al. examined the effects of an eight week, 16 session hospital-based program consisting of both aerobic exercise and weight training. The aerobic part of the training session involved exercising on a treadmill and a bike. Strength training consisted of six exercises. It was found that a structured exercise program is feasible for advanced non-small cell lung cancer patients [25].
The concept of HIIT is that periods of high intensity training are alternated with low intensity recovery periods. This allows the participant to spend more time in high-intensity zones, producing a stronger stimulus for muscle development including capillary density and blood flow as well as mitochondrial density and improvements in cardiovascular function [10]. The HIIT protocol resulted in a marked improvement in the amount of power output across the intervention period and the amount of yielded power performed more than doubled (100\% improvement) over the training period. RPE remained relatively constant throughout training sessions, which suggests that participants were capable of maintaining an elevated exercise intensity at every training session. This shows that while average training volume increased, participants maintained the same level of effort while performing higher training volumes, suggesting that participants were capable of maintaining an elevated exercise intensity at every training session.

Stage IIIB and IV NSCLC tumors are typically very difficult to remove if not impossible, making a cure very unlikely. Stage IV NSCLC involves metastasis of the original tumor and more than $50 \%$ of lung cancer diagnoses are stage IV [26]. Treatment typically includes chemotherapy, immunotherapy, and/or radiation [27]. Nine of our participants were diagnosed with stage IV NSCLC and one was diagnosed with stage IIIB NSCLC initially. After the end of the six weeks, the stage IIIB participant developed bone metastases to his lumbar spine and progressed to stage IV. These two types of NSCLC cannot typically be cured, but they can be treated and exercise may be a helpful option for these patients to improve quality of life. 


\section{Limitations}

There were some limitations to this study. One of the limitations is its small sample size. Further studies with a larger sample size are necessary to explore the effects of HIIT in NSCLC patients. Exercise type performed at home was not controlled, meaning that we were not able to quantify the amount of exercise performed by control patients. A 6-week intervention remains short to observe significant changes in participants and further studies examining a longer intervention would be beneficial. Nonetheless, a longer duration program may increase the dropout rate.

\section{Conclusion}

In conclusion, advanced NSCLC patients are able to do a HIIT program. They show progression in intensity and number of intervals. RPE remained relatively constant, indicating that maximum sustainable effort was maintained at every training session. Thus, the application of HIIT as part of the overall treatment plan for advanced NSCLC patients seems feasible, is more exciting and takes less time than a standard exercise program while providing the same benefits and more interestingly, shows muscle power improvement in a short period of time.

\section{Conflicts of Interest}

None.

\section{Authorship Contribution}

EW and GB- collected and analyzed the data, supervised performance of each HIIT session; EWcomposed the first draft; GHB- reviewed and edited the manuscript and added scientific contributions; AFrevised the manuscript and edited scientific content; ASC-revised the final draft, edited scientific content, and is the guarantor of the paper.

\section{References}

1. Siegel RL, Miller KD, Fuchs HE, Jemal A (2021) Cancer statistics CA: A Cancer Journal for Clinicians 71: 7-33.

2. Cancer Facts \& Figures (2021) American Cancer Society.

3. Arem H, Moore SC, Park Y, Ballard-Barbash R, Hollenbeck A, et al. (2014) Physical activity and cancer-specific mortality in the NIH-AARP Diet and Health Study cohort. Int J Cacer 135: 423- 431.

4. Koutsokera A, Kiagia M, Saif MW, Souliotis K, Syrigos KN (2013) Nutrition habits, physical activity, and lung cancer: An authoritative review. Clin Lung Cancer 14: 342-350.

5. Brown JC, Winters-Stone K, Lee A, Schmitz KH (2012) Cancer, Physical Activity, and Exercise. Comp Physiol 2: 2775-2809.

6. Douglas E (2005) Exercise in cancer patients. Physical Therapy Reviews 10: 71-88.

7. Oechsle K, Aslan Z, Suesse Y, Jensen W, Bokemeyer C, et al. (2014) Multimodal exercise training during myeloablative chemotherapy: A prospective randomized pilot trial. Support Care Cancer 22: 63-69.

8. Granger CL, Denehy L, McDonald CF, Irving L, Clark RA (2014) Physical activity measured using global positioning system tracking in non-small cell lung cancer: An observational study. Integrative Cancer Therapies 13: 482-492.

9. Laursen P, Jenkins D (2002) The scientific basis for highintensity interval training: Optimising training programmes and maximising performance in highly trained endurance athletes. Sports Med 32: 53-73.

10. Meyer P, Gayda M, Juneau M, Nigam A (2013) Highintensity aerobic interval exercise in chronic heart failure. Curr Heart Fail Rep 10: 130-138.

11. Wallen MP, Hennessy D, Brown S, Evans L, Rawstorn JC, et al. (2020) High-intensity interval training improves cardiorespiratory fitness in cancer patients and survivors: A meta-analysis. European journal of cancer care 29: 13267.

12. Adams SC, DeLorey DS, Davenport MH, Fairey AS, North S, et al. (2018) Effects of high- intensity interval training on fatigue and quality of life in testicular cancer survivors. $\mathrm{Br}$ JCancer 118: 1313-1321.

13. Mijwel S, Backman M, Bolam KA, Jervaeus A, Sundberg CJ, et al. (2018) Adding high-intensity interval training to conventional training modalities: Optimizing health-related outcomes during chemotherapy for breast cancer: The OptiTrain randomized controlled trial. Breast Cancer Res Treat 168: 79-93.

14. Licker M, Karenovics W, Diaper J, Schorer R, Kayser B, et al. (2017) Short-term preoperative high-intensity interval training in patients awaiting lung cancer surgery: A Randomized controlled trial. Journal of thoracic oncology 12: 323-333.

15. Kravitz L (2011) High-intensity interval training. American College of Sports Medicine 1-2.

16. American thoracic society statement: Guidelines for the sixminute walk test. American Thoracic Society.

17. Perceived exertion (borg rating of perceived exertion scale). CDC Website.

18. Takai $Y$, Ohta M, Akagi R, Kanehisa $H$, Kawakami $Y$, et al. (2009) Sit-to- stand Test to Evaluate Knee Extensor Muscle Size and Strength in the Elderly: A Novel Approach. Journal of Physiological Anthropology 28: 123-128.

19. Kalinova E, Leone M (2009) Tests d'evaluation de la capacite fonctionnelle. Quebec: Presses de la l' Universite du Quebec.

20. Guiraud T, Nigam A, Gremeaux V, Meyer $P$, Juneau M, et al. (2012) High- Intensity Interval Training in Cardiac Rehabilitation. Sports Med 42: 587-604.

21. Weston KS, Wisløff U, Coombes JS (2014) Highintensity interval training in patients with lifestyle-induced cardiometabolic disease: A systematic review and metaanalysis. Br J Sports Med 48: 1227-1234.

22. Andersen AH, Vinther A, Poulsen L, Mellemgaard (2011) Do patients with lung cancer benefit from physical exercise? Acta Oncol 50: 307-313.

23. Bartlett JD, Close GL, Maclaren DPM, Gregson W, Drust $B$, et al. (2011) High-intensity interval running is perceived to be more enjoyable than moderate-intensity continuous exercise: Implications for exercise adherence. J Sports Sci 29: 547-553.

24. Hwang C, Yu C, Shih J, Yang P, Wu Y (2012) Effects of exercise training on exercise capacity in patients with nonsmall cell lung cancer receiving targeted therapy. Support Care Cancer 20: 3169-3177.

25. Temel JS, Greer JA, Goldberg S, et al. (2009) NIH Public Access 4: 595-601.

26. Cancer stat facts: Lung and bronchus cancer. National Cancer Institute.

27. Lung cancer stages. Cancer Treatment Centers of America. 